\title{
Gastrointestinal Hemorrhage in Left Ventricular Assist Device Patients: Red Scare or Pressure from the Right?
}

\author{
Grigoriy E. Gurvits ${ }^{1}$
}

Published online: 27 March 2018

(c) Springer Science+Business Media, LLC, part of Springer Nature 2018

Rapid technological progress over the last quarter of the twentieth century has paved the way to many of the breakthrough advances in medical field. Among the most remarkable innovations are implantable appliances designed to improve the survival of some of the sickest patients. Affecting nearly 6 million Americans and extending to $10 \%$ of the geriatric population [1], congestive heart failure (CHF) was traditionally managed solely with pharmacological therapy until various mechanical supports-first external and pneumatic and later internal and electric_-gradually appeared in clinical practice. The landmark 2001 randomized evaluation of mechanical assistance of congestive heart failure (REMATCH) trial [2] brought left ventricular assist devices (LVAD) into the international spotlight by showing their efficacy and safety, effectively gaining acceptance of the LVAD as the standard-of-care for end-stage heart failure patients both as bridge to transplant and as a long-term destination therapy in cases where heart transplant is not an option [3]. Use of pulsatile and, later, continuous flow models has exponentially grown in the USA from 238 (in 2007) to 1771 (in 2011) to 2884 LVADs (in 2015) [4] as many tertiary care centers initiated their own LVAD programs. The reported number of implanted devices has reached nearly 20,000 over the 11-year period ending in 2017 [4]. Increased utilization of the device and its clinical and technological improvement prolonged survival and improved the quality-of-life for many recipients, challenging some of the early concerns and increasing its popularity among patients and medical practitioners.

With all the press glamour and their positive effect on the functional status of end-stage heart failure patients, LVADs do come with a variety of adverse effects and complications.

Grigoriy E. Gurvits

g_gurvits@hotmail.com

1 Division of Gastroenterology, New York University School of Medicine/Langone Medical Center, 555 Madison Avenue, 3 Fl, New York, NY 10022, USA
Historically, up to $60 \%$ of LVAD recipients may develop gastrointestinal (GI) hemorrhage [5] —an important issue given increased prevalence of implantation worldwide. In fact, it is the most common adverse event in patients with post-device implantation [3] and typically resolves after a heart transplant $[5,6]$. Clinical effects of bleeding may vary, ranging from continual anemia in some patients to overt hemorrhage with hemodynamic compromise in others. Its etiology is largely multifactorial: a combination of predisposing medical conditions, systemic and intrinsic properties of the devices, associated antiplatelet and anticoagulation therapy, and endogenous fibrinolysis. Since its inception and effective clinical use, much medical research has been published on better understanding of the processes involved and managing GI hemorrhage without compromising the risk of thromboembolic disease in LVAD patients. In this respect, the paper by Dr. Tomizawa and colleagues from the University of Chicago Medical Center published in this issue of Digestive Diseases and Sciences [7] highlights the importance of risk stratification of pre-implant patients and attempts to define additional factors associated with the post-implant GI bleeding. The authors illustrate that preoperative right-sided cardiac congestion is an independent predictor for GI hemorrhage in patients implanted with continuous flow LVAD.

Although this observation is not novel, the current study adds to the growing evidence that heart failure and specifically advanced right-sided dysfunction affect a variety of intrinsic hemostatic pathways. There is an inherent low-level auto-anticoagulation in end-stage heart failure patients [8]. Platelet hypersensitivity and degranulation are also present and result in relative dysfunction that is further potentiated by uremia in many individuals with coexistent renal hypoperfusion. Additionally, activation of fibrinolytic systems is notable in older patients prior to LVAD placement, likely a result of preexisting state of inflammation [9]. Finally, recent literature indicates that right ventricular dysfunction and post-LVAD ejection 
fraction $>30 \%$ are associated with higher incidence of GI hemorrhage in device recipients [10]—an important observation in this group of patients that is probably related to the notable angioectasia formation in the proximal GI tract. The routine use of antiplatelet agents (aspirin) and anticoagulants (warfarin) for stroke prevention, acquired von Willebrand factor (vWF) syndrome (loss of high molecular weight vWF multimers required for platelet aggregation) following LVAD placement substantially potentiates the risk of bleeding, but there ultimately needs to be a lesion in the GI tract for such a hemorrhage to occur. Chronic right-sided congestion combined with postLVAD increased cardiac output into a narrow pulse-pressure system in close proximity to the celiac axis and proximal jejunal branches of the superior mesenteric artery may be responsible for pathogenesis of the friable angioectasias in the upper GI tract. Indeed, no patient in Dr. Tomizawa's study had history of GI bleeding prior to LVAD implantation, an important concept in the discussion of the study results.

The crucial notion of the small intestinal angioectatic bleeding is central in managing such patients. While the conventional approach with endoscopy and colonoscopy may be employed in the initial evaluation of the cause of GI blood loss in LVAD recipients, the use of endoscopic tools to examine small intestine, with particular attention to the area in proximity of the ligament of Treitz, is essential to establishing the correct diagnosis and administering appropriate therapeutic interventions. Indeed, angioectasias account for the majority of the culprit lesions, with particular predilection to the upper GI tract. We have previously shown that routine use of push enteroscopy in LVAD patients with GI hemorrhage increased diagnostic yield threefold, from 29 to $90 \%$, successfully eradicating lesions and achieving endoscopic hemostasis, potentially decreasing future GI readmission rates $[6,11]$. Such an algorithm has been subsequently accepted at the institutional level and remains an effective tool in clinical practice. Other endoluminal modalities include small bowel video capsule endoscopy that may be helpful in determining the locus of occult bleeding in the outpatient setting and/or examining the small bowel outside the reach of push enteroscope. Single- and double-balloon advanced enteroscopy are additional tools in gastroenterologist's armamentarium that may subsequently be employed for endoluminal therapy of mid-distal small bowel lesions. It would certainly be interesting to extrapolate these data from Dr. Tomizawa's study, but given its retrospective design, as the authors mentioned, only one-third of patients were referred for the small intestinal assessment. Future studies directed at evaluation of the GI tract and specifically the small bowel prior to and after LVAD implantation may ultimately lead to a better understanding of the pathophysiology, anatomy, and risk stratification of GI hemorrhage in such patients. Any insights gained would translate into decreased cumulative cost and improved patient outcomes associated with LVAD implantation.

Finally, Dr. Tomizawa and colleagues raised the important issue of formulating a primary prevention strategy in patients undergoing LVAD placement. The authors show that preoperative right-sided congestion may place LVAD recipients at increased risk of developing postoperative hemorrhage. Previous studies have suggested that the lowest chance of bleeding occurred in blood type A patients [12] and the highest in patients with history of GI hemorrhage prior to LVAD placement [11]. Furthermore, male gender and older age are recognizable risk factors [5]. There also seems to exist a possible correlation with GI hemorrhage in patients with easily observed nasal hypervascularity following LVAD implantation [13]. Additional variables will continue to emerge with increased utilization of the devices and associated interest in further clinical research. Prospective medical therapies tailored to account for recognized individual predispositions to GI bleeding may ultimately decrease complication rates in LVAD recipients. Platelet function and aggregation studies may prove to be beneficial. Potential use of desmopressin, similar to its implementation in von Willebrand disease phenotype 2a, may gain acceptance in the future. Similarly, development of angioectasias may possibly be offset by the early use of octreotide-a somatostatin analog that decreases splanchnic blood flow and inhibits angiogenesis. Variable device speed regulation, enhancement of biomaterial, and adjustable coagulation parameters may gain additional recognition and clinical application as well. As such, today's multidisciplinary approach with cooperation among the cardiology, gastroenterology, and hematology services would factor in important aspects of each discipline, ultimately enhancing the prevention and management of future GI complications following LVAD placement in CHF patients.

\section{Compliance with ethical standards}

Conflict of interest None.

\section{References}

1. Heidenreich PA, Albert NM, Allen LA, et al. Forecasting the impact of heart failure in the United States: a policy statement from the American Heart Association. Circ Heart Fail. 2013;6:606-619.

2. Rose EA, Gelijns AC, Moskowitz AJ, et al. Long term use of left ventricular assist device for end-stage heart failure. $N$ Engl J Med. 2001;345:1435-1443.

3. Tsiouris A, Paone G, Nemeh HW, et al. Short and long term outcomes of 200 patient supported by continuous-flow left ventricular assist device: a case series. World J Cardiol. 2015;7:792-800. 
4. INTERMACS Interagency Registry for Mechanically Assisted Circulatory Supports quarterly statistical report. Implant dates: June 23, 2006-Sept 30, 2017. Available from: https://www.uab. edu/medicine/intermacs/images/Federal_Quarterly_Report/Feder al_Partners_Report_2017_Q3.pdf [2017 Dec 18; cited 2018 Feb 5].

5. Gurvits GE, Fradkov E. Bleeding with the artificial heart: gastrointestinal hemorrhage in CF-LVAD patients. World J Gastroenterol. 2017;23:3945-3953.

6. Stern DR, Kazam J, Edwards P, et al. Increased incidence of gastrointestinal bleeding following implantation of the HeartMate II LVAD. J Card Surg. 2010;25:352-356.

7. Tomizawa Y, Tanaka A, Kitahara H, et al. Preoperative RightSided Cardiac Congestion is Associated with gastrointestinal Bleeding in Patients with Continuous-Flow Left Ventricular Assist Devices.Dig Dis Sci (Epub ahead of print). https://doi. org/10.1007/s10620-018-4916-8.

8. Slaughter MS, Sobieski MA, Gallagher C, et al. Fibrinolytic activation during long-term support with the HeartMate II left ventricular assist device. ASAIO J. 2008;54:115-119.
9. Slaughter MS. Hematologic effects of continuous flow left ventricular assist devices. J CardiovascTransl Res. 2010;3:618-624.

10. Jabbar HR, Abbas A, Ahmed M, et al. The incidence, predictors and outcomes of gastrointestinal bleeding in patients with Left Ventricular Assist Device (LVAD). Dig Dis Sci. 2015;60:36973706. https://doi.org/10.1007/s10620-015-3743-4.

11. Marsano J, Desai J, Chang S, et al. Characteristics of gastrointestinal bleeding after placement of continuous-flow left ventricular assist device: a case series. Dig Dis Sci. 2015;60:1859-1867. https ://doi.org/10.1007/s10620-015-3538-7

12. Crow S, Chen D, Milano C, et al. Acquired von Willebrand syndrome in continuous-flow ventricular assist device recipients. Ann ThoracSurg. 2010;90:1263-1269.

13. Patel SR, Saeed O, Madan S, et al. Vascular abnormalities of the nasal mucosa are associated with gastrointestinal bleeding during continuous flow LVAD support. J Heart Lung Transpl. 2016;35:S84. 